\title{
Cost analysis of medical assistance in dying in Canada
}

\author{
Aaron J. Trachtenberg MD DPhil, Braden Manns MD MSc
}

Cite as: CMAJ 2017 January 23;189:E101-5. doi: 10.1503/cmaj.160650

See also www.cmaj.ca/lookup/doi/10.1503/cmaj.161316

\begin{abstract}
BACKGROUND: The legalization of medical assistance in dying will affect health care spending in Canada. Our aim was to determine the potential costs and savings associated with the implementation of medical assistance in dying.
\end{abstract}

METHODS: Using published data from the Netherlands and Belgium, where medically assisted death is legal, we estimated that medical assistance in dying will account for $1 \%-4 \%$ of all deaths; $80 \%$ of patients will have cancer; $50 \%$ of patients will be aged $60-80$ years; $55 \%$ will be men; $60 \%$ of patients will have their lives shortened by 1 month; and $40 \%$ of patients will have their lives shortened by 1 week. We combined current mortality data for the Canadian population with recent endof-life cost data to calculate a predicted range of savings associated with the implementation of medical assistance in dying. We also estimated the direct costs associated with offering medically assisted death, including physician consultations and drug costs.

RESULTS: Medical assistance in dying could reduce annual health care spending across Canada by between $\$ 34.7$ million and $\$ 138.8$ million, exceeding the $\$ 1.5-\$ 14.8$ million in direct costs associated with its implementation. In sensitiv- ity analyses, we noted that even if the potential savings are overestimated and costs underestimated, the implementation of mdedical assistance in dying will likely remain at least cost neutral.

INTERPRETATION: Providing medical assistance in dying in Canada should not result in any excess financial burden to the health care system, and could result in substantial savings. Additional data on patients who choose medical assistance in dying in Canada should be collected to enable more precise estimates of the impact of medically assisted death on health care spending and to enable further economic evaluation.
$\mathrm{T}$ he Criminal Code of Canada's prohibitions on medical assistance in dying have been lifted. ${ }^{1}$ The logistics of offering medical assistance in dying to Canadians need to be formalized, and the consequences of this practice need to be anticipated. As with any new medical intervention, understanding the clinical and costing implications of medical assistance in dying is important.

Health care costs increase substantially among patients nearing the end of life, accounting for a disproportionate amount of health care spending. For example, in Manitoba, more than $20 \%$ of health care costs are attributable to patients within the 6 months before dying, despite their representing only $1 \%$ of the population. ${ }^{2}$ Furthermore, as death approaches, health care costs increase dramatically in the final months. ${ }^{3,4}$ Patients who choose medical assistance in dying may forego this resource-intensive period.

In a 1998 special article in The New England Journal of Medicine, Emanuel and Battin predicted that legalizing medical assistance in dying in the United States would save hundreds of millions of health care dollars per year,; a similar model can be applied to Canada. Here, we combine data on the use of medical assistance in dying from countries where it is legal with Canadian-specific end- of-life cost data to estimate the effect of this intervention on health care costs in Canada.

\section{Methods}

Emanuel and Battin's model relies on 3 main factors to estimate cost savings from medical assistance in dying: the number of patients expected to choose the intervention; the effect of the intervention on life expectancy; and the total health care costs associated with end-of-life care. ${ }^{5}$ We updated their model to include more recent and detailed demographic estimates of the patients who may choose medical assistance in dying, including age, sex and underlying diagnosis, in addition to Canadian-specific cost data.

\section{Proportion of deaths due to medical assistance} in dying and patient demographics

Between 1990 and 2012, medical assistance in dying accounted for an estimated $1.8 \%-3.3 \%$ of total deaths in the Netherlands, with a trend for an increase in rates over the last 25 years. ${ }^{6,7}$ In Belgium, medical assistance in dying has accounted for between 
$0.3 \%$ and $4.6 \%$ of all deaths, with the highest rates documented in the most recent 2013 survey. ${ }^{8,9}$ Based on these numbers, we estimated that medical assistance in dying will eventually play a role in $1 \%-4 \%$ of all deaths in Canada.

Several individual studies, in addition to a recent systematic review, have shown that medical assistance in dying is most commonly chosen by men, by patients in their 7th and 8th decade of life and by patients with cancer. ${ }^{6,10,11}$ Based on these previous data, we assumed that $55 \%$ of patients choosing the intervention will be male, that $50 \%$ of patients will be aged 60-79 years, $30 \%$ will be aged $18-59$ years and $20 \%$ will be 80 years and older, and that $80 \%$ of patients will have cancer.

\section{Impact on life expectancy}

A 2010 physician survey from the Netherlands estimated that $40 \%$ of cases of medical assistance in dying were associated with a reduction in life expectancy of less than 1 week, and 60\% were associated with a reduction of more than 1 week (no upper time limit provided). ${ }^{6}$ Thus, we predicted that about $40 \%$ of Canadians who choose medical assistance in dying will have their lives shortened by 1 week, and $60 \%$ of patients will have their lives shortened by 1 month.

\section{Cost of end-of-life care}

With the collaboration of the authors (and after ethics approval), previously published costing data from the province of Ontario ${ }^{3}$ were reanalyzed into the relevant subgroups for our model. Briefly, total health care costs for all decedents in Ontario between Apr. 1, 2010, and Mar. 31, 2013 ( $n=264755$ ), were measured using administrative data. These costs were calculated at an individual patient level on a cumulative daily basis, which allowed for weekly and monthly costs preceding death to be determined. Costs included those associated with acute care (emergency department visits and hospital admissions), outpatient care (physician billing, outpatient clinics [including dialysis and cancer care], laboratory services and select drug or device expenses) and continuing care (long-term care, complex continuing care, rehabilitation and home care). Additional details are available in the original publication. ${ }^{3}$

We excluded deaths from "external causes" (accident, poisoning, assault, drowning, and complications of medical care, as defined by International Statistical Classification of Diseases and Related Health Problems, 10th revision [ICD-10], codes). Patients were considered to have cancer if they had 1 hospital admission with a discharge diagnosis or 2 outpatient visits with an ICD-10 billing code of neoplasm in their final 2 years of life. We created subgroups according to sex and age, as defined previously, and calculated the average total cost of health care use in the final month and final week of life for each subgroup.

\section{Estimating potential savings associated with medical assistance in dying}

We assumed 268056 total deaths annually in Canada based on Statistics Canada figures for the period July 1, 2014, to June 30, $2015 .{ }^{12}$ We ran 4 iterations of our model in which medical assistance in dying accounted for $1 \%, 2 \%, 3 \%$ and $4 \%$ of total deaths.
We used the total number of cases to derive the number of patients in each subgroup using the assumptions described previously.

We then multiplied the number of cases in each subgroup by the mean total cost of either the final week or final month of life associated with that subgroup. We combined the total cost associated with each subgroup to estimate the potential cost savings in a given year associated with medical assistance in dying.

\section{Effect on costs with the assumption that patients selected palliative care}

Our base-case model uses mean costs for all decedent subgroups, which can be interpreted as the cost associated with standard end-of-life care across a variety of scenarios and patient wishes. It is possible that patients who request medical assistance in dying would choose a less aggressive palliative care approach. Because a previous review suggested that palliative care reduces end-of-life health care costs by $40 \%-70 \%$ compared with standard care, ${ }^{13}$ we conducted 2 additional analyses in which we reduced the cost of care preceding death by $40 \%$ and $70 \%$.

\section{Additional sensitivity analyses}

We conducted additional sensitivity analyses using our base-case analysis (assuming 1\% of decedents select medical assistance in dying) varying our assumptions regarding sex, age group, cancer status and effect on life expectancy. The variations we modelled included $30 \%-70 \%$ of cases involving male patients, $50 \%-100 \%$ of cases involving cancer patients, equal division of cases among the 3 age groups, and plausible variations in the proportion of patients who may have their life expectancy reduced by 1 month as opposed to 1 week.

\section{Estimating the costs associated with implementing medical assistance in dying}

There is no standardized approach to medical assistance in dying yet in Canada, and each case will have unique patient and provider elements, which makes estimating the costs associated with this service difficult. Acknowledging this, we estimated the direct costs of offering medical assistance in dying within a fee-for-service setting, understanding that there will have to be 2 independent assessments for patient eligibility, ${ }^{14}$ and that medications chosen by the physician involved will be administered at a separate visit after being prepared by a pharmacy. Therefore, each case will include at least 2 physician claims for a consultation, 1 claim for a follow-up visit where the medication is administered, the cost of the drugs in addition to pharmacy preparation time and 1 claim for completion of the death certificate. To keep costs consistent and comparable with our end-of-life costing analysis, we used Ontario physician fees in our calculations. ${ }^{15}$ Although there is no standard drug regimen for medical assistance in dying across Canada, we had confidential access to the drug regimens suggested in Alberta (including an anxiolytic, local anesthetic, sedative and paralytic), as well as the associated costs. Costs for these alternate regimens varied between $\$ 25$ and $\$ 326$ per case. We assumed 
an additional $\$ 30$ of pharmacist time (about $30 \mathrm{~min}$ of time to prepare and dispense the drugs based on a mean pharmacist wage of $\$ 60 / \mathrm{h}$, including benefits ${ }^{16}$ ).

We created 2 base-case scenarios, a low-cost scenario in which the assessments are performed by primary care physicians and the least costly drug regimen is used, and a high-cost scenario in which the assessments are done by specialists and the most costly drug regimen is used (Table 1 ). In addition, there will be patients who request medical assistance in dying and incur the 2 assessment fees but do not complete the process, either being denied, retracting their request or dying from their disease. In the Netherlands, data from a single centre suggests $25 \%$ of requests for medical assistance in dying are ultimately granted, ${ }^{17}$ whereas this proportion is more than $75 \%$ in Belgium. ${ }^{18}$ We chose a conservative estimate that $33 \%$ of requests in Canada will be granted, meaning for every patient receiving medical assistance in dying, we added the cost of 2 additional patients who underwent assessment only.

\section{Results}

Table 2 shows the mean health care spending in the final week and final month of life that was used to calculate potential cost savings for each subgroup. The potential cost of implementing medical assistance in dying, and the estimated savings in end-oflife care associated with the intervention, are shown in Table 3. Assuming a low-cost scenario and standard end-of-life care (i.e., base-case analysis), we expect that net health care costs would be reduced by $\$ 33.2$ million per year if $1 \%$ of deaths are due to medical assistance in dying. When end-of-life costs were reduced by $40 \%$ and $70 \%$ with the assumption that a palliative approach would have been chosen, net cost savings are reduced to $\$ 19.3$ million and $\$ 8.9$ million, respectively (Table 3). As noted within Table 3, overall health care costs are lower across all scenarios considered, even when higher drug and physician costs are assumed.

Varying the sex, cancer status and age group of the patients across plausible ranges had little effect on the projected savings, whereas varying the estimated effect on life expectancy had a larger impact on projected savings. For example, if $80 \%$ of patients have their lives shortened by 1 month (rather than $1 \mathrm{wk}$ ), there would be an additional $\$ 5.7$ million in health care savings, whereas if $80 \%$ of patients have their life expectancy shortened by only 1 week, the projected savings would be reduced by $\$ 11.3$ million, or more than $30 \%$.

\section{Table 1: Inputs used to calculate the costs associated with} implementing medical assistance in dying in Canada

\begin{tabular}{lc} 
Input* & Cost, \$ \\
\hline Low-cost scenario & \\
\hline Primary care physician assessment & 77.20 \\
\hline Primary care physician follow-up visit & 38.35 \\
\hline Medications & 25.40 \\
\hline Pharmacist time & 30.00 \\
\hline Completion of death certificate & 20.60 \\
\hline Total for completed case & 268.75 \\
\hline Total for assessed case & 154.40 \\
\hline High-cost scenario & \\
\hline Internal medicine specialist assessment & 157.00 \\
\hline Internal medicine follow-up visit & 61.25 \\
\hline Medications & 326.00 \\
\hline Pharmacist time & 30.00 \\
\hline Completion of death certificate & 20.60 \\
\hline Total for completed case & 751.85 \\
\hline Total for assessed case & 314.00 \\
\hline *Costs based on Ontario Schedule of Benefits, & 15 \\
costs, which are based on Alberta protocols. ${ }^{*}$ & \\
\hline
\end{tabular}

Table 2: Expected number of cases of medical assistance in dying and associated health care spending in the final week and month of life among men and women with and without cancer*

\begin{tabular}{|c|c|c|c|c|c|c|c|c|}
\hline \multirow[b]{3}{*}{ Age, yr } & \multicolumn{4}{|c|}{ Expected death within 1 wk } & \multicolumn{4}{|c|}{ Expected death within 1 mo } \\
\hline & \multicolumn{2}{|r|}{ Men } & \multicolumn{2}{|c|}{ Women } & \multicolumn{2}{|r|}{ Men } & \multicolumn{2}{|c|}{ Women } \\
\hline & No. & Mean cost, \$ & No. & Mean cost, \$ & No. & Mean cost, \$ & No. & Mean cost, \$ \\
\hline \multicolumn{9}{|c|}{ With cancer $(n=4289)$} \\
\hline $18-59$ & 283 & 7430 & 232 & 6897 & 425 & 19958 & 347 & 19115 \\
\hline $60-79$ & 472 & 6880 & 386 & 6420 & 708 & 17996 & 579 & 17608 \\
\hline$\geq 80$ & 188 & 5725 & 154 & 4980 & 283 & 14846 & 232 & 13236 \\
\hline \multicolumn{9}{|c|}{ Without cancer ( $n=1072$ ) } \\
\hline $18-59$ & 71 & 7090 & 58 & 8293 & 106 & 14673 & 87 & 18063 \\
\hline $60-79$ & 118 & 7144 & 97 & 7080 & 177 & 16470 & 145 & 16450 \\
\hline$\geq 80$ & 47 & 5264 & 39 & 4065 & 71 & 12861 & 58 & 10253 \\
\hline
\end{tabular}

*Numbers were derived with the assumption that medical assistance in dying would account for $2 \%$ of total deaths in Canada ( $n=268056$ from July 1,2014 , to June 30,2015 ) and that $80 \%$ of patients would have cancer. The sum of numbers in each subgroup may be greater than the total because of rounding. 


\section{Interpretation}

If Canadians adopt medical assistance in dying in a manner and extent similar to those of the Netherlands and Belgium, we can expect a reduction in health care spending in the range of tens of millions of dollars per year. Our analyses suggest that the savings will almost certainly exceed the costs associated with offering medical assistance in dying to patients across the country, and that the inclusion of medical assistance in dying in the services covered by universal health care will not increase health care spending, but rather will release funds to be reinvested elsewhere. We are not suggesting medical assistance in dying as a measure to cut costs. At an individual level, neither patients nor physicians should consider costs when making the very personal decision to request, or provide, this intervention.

\section{Strengths and limitations}

We used comprehensive Canadian-specific cost data, provided a range of potential savings based on projected uptake of medical assistance in dying across the country, used more detailed subgroupings to create case estimates than in a previously published American model, ${ }^{5}$ and performed extensive sensitivity analyses. We provided a preliminary estimate of the direct costs of medical assistance in dying in Canada, including the costs of requests that are not ultimately granted.

The main limitation of our model was using data from the Netherlands and Belgium to build our case estimates. In Canada, only competent adults aged 18 years and older whose "natural death has become reasonably foreseeable" will be eligible for medical assistance in dying, ${ }^{14}$ whereas in the Netherlands and Belgium, there are provisions to allow medical assistance in dying for patients with nonterminal diseases, minors and patients with dementia. ${ }^{19,20}$ Therefore, we may have overestimated the propor- tion of total deaths due to medical assistance in dying by using statistics from countries with more liberal policies. We did, however, provide a range of estimates to account for this possibility.

Our cost data were specific to the province of Ontario, and may not represent end-of-life spending in all Canadian provinces. ${ }^{3}$ However, these are the most comprehensive data available on end-of-life health care costs in Canada, including costs across a broader range of services (such as outpatient visits and diagnostic investigations) than previous reports. ${ }^{2,4}$ In addition, these cost data are recent and therefore more likely to reflect current fee schedules and current standards of care at the endof-life, including new treatments. Finally, the costs were calculated on a daily basis, allowing for the use of weekly and monthly subtotals, which were crucial to our model.

It should be noted that our estimates regarding the effect of medical assistance in dying on life expectancy were taken from a single physician survey. ${ }^{6}$ Because our sensitivity analyses found this variable to have a considerable effect on our results, determining how to accurately measure and report this information for Canadian cases will be important for future cost analyses.

Our estimates of the costs associated with offering medical assistance in dying were based on a very simple model, which predominantly used Ontario-level costs for consistency. This analysis should be updated if and when unique billing codes for the consultations and delivery of medical assistance in dying are developed. We did not account for additional potential costs, such as coordination services. In Alberta, there is a medical assistance in dying coordination team with several full-time employees who respond to patient or provider inquiries and assist in liaising patients with willing providers. These are fixed costs, and do not necessarily vary with the number of cases, but could be substantial if every province adopts this approach. Administrative costs associated with creating an oversight body or reporting mechanism, as suggested by the

Table 3: Estimated health care costs and savings associated with medical assistance in dying in Canada

$\%$ of total deaths due to medical assistance in dying $(n)$

\begin{tabular}{|c|c|c|c|c|}
\hline Cost and savings & $1 \%(2681), \$$ & $2 \%(5361), \$$ & $3 \%(8042), \$$ & $4 \%(10722), \$$ \\
\hline \multicolumn{5}{|l|}{ Gross savings } \\
\hline Base-case: mean end-of-life costs & 34706110 & 69412219 & 104118329 & 138824438 \\
\hline Palliative care scenario: $40 \%$ reduction in end-of-life costs & 20823666 & 41647331 & 62470997 & 83294662 \\
\hline Palliative care scenario: $70 \%$ reduction in end-of-life costs & 10411833 & 20823666 & 31235499 & 41647331 \\
\hline \multicolumn{5}{|l|}{ Cost of providing medical assistance in dying } \\
\hline Low-cost scenario & 1548412 & 3096246 & 4644657 & 6192491 \\
\hline High-cost scenario & 3699378 & 7397376 & 11096754 & 14794752 \\
\hline \multicolumn{5}{|l|}{ Net savings } \\
\hline Base-case - low-cost scenario & 33157698 & 66315974 & 99473671 & 132631947 \\
\hline Base-case - high-cost scenario & 31006732 & 62014843 & 93021575 & 124029686 \\
\hline Palliative $40 \%$ reduction in end of life cost - low-cost scenario & 19275254 & 38551085 & 57826340 & 77102171 \\
\hline Palliative $40 \%$ reduction in end of life cost - high-cost scenario & 17124288 & 34249955 & 51374243 & 68499910 \\
\hline Palliative $70 \%$ reduction in end of life cost - low-cost scenario & 8863421 & 17727420 & 26590842 & 35454840 \\
\hline Palliative $70 \%$ reduction in end of life cost - high-cost scenario & 6712455 & 13426290 & 20138745 & 26852579 \\
\hline
\end{tabular}


Canadian Medical Association, ${ }^{21}$ were also not accounted for. Nevertheless, even if these costs were included, it is likely that the implementation of medical assistance in dying would be cost neutral or result in cost savings.

Finally, our analysis is only a cost analysis and it does not consider the clinical effects of medical assistance in dying on patients at the end of life. Patient-level research that explores the reasons why Canadians choose medical assistance in dying, the value they assign to their suffering versus death and other aspects of their experience will need to be done before true economic evaluation of medical assistance in dying in terms of costeffectiveness and utility can be done.

\section{Conclusion}

Our analysis suggests that the provision of medical assistance in dying in Canada will be cost neutral or result in a reduction in total health care costs, although the true effect on health care costs will not be certain until we determine who the typical Canadian patient requesting the intervention is and how its practice is implemented across the country. Our study highlights the need to prospectively collect certain data to accurately measure the effect of this new policy on health care spending, namely the total number of patients, in addition to demographics, underlying diagnosis and estimated effect on life expectancy.

The implementation of medical assistance in dying is a major event in Canadian history. Anticipating, measuring and responding to the broad range of effects that may result from this decision should be considered the responsibility of physicians, politicians and policy-makers alike.

\section{References}

1. Carterv. Canada (Attorney General). 2016 SCC 4. Ottawa: Supreme Court of Canada.

2. Menec V, Lix L, Steinbach C, et al. Patterns of health care use and cost at the end of life. Winnipeg: Manitoba Centre for Health Policy; 2004.

3. Tanuseputro P, Wodchis WP, Fowler R, et al. The health care cost of dying: a population-based retrospective cohort study of the last year of life in Ontario, Canada. PLoS One 2015;10:e0121759.
4. Health care use at the end of life in Saskatchewan. Ottawa: Canadian Institute for Health Information; 2008.

5. Emanuel EJ, Battin MP. What are the potential cost savings from legalizing physician-assisted suicide. N Engl J Med 1998;339:167-72.

6. Onwuteaka-Philipsen BD, Brinkman-Stoppelenburg A, Penning C, et al. Trends in end-of-life practices before and after the enactment of the euthanasia law in the Netherlands from 1990 to 2010: a repeated cross-sectional survey. Lancet 2012;380:908-15.

7. Lerner BH, Caplan AL. Euthanasia in Belgium and the Netherlands: On a slippery slope? JAMA Intern Med 2015;175:1640-1.

8. Chambaere K, Bilsen J, Cohen J, et al. Trends in medical end-of-life decision making in Flanders, Belgium 1998-2001-2007. Med Decis Making 2011;31:500-10.

9. Chambaere K, Vander Stichele R, Mortier F, et al. Recent trends in euthanasia and other end-of-life practices in Belgium. N Engl J Med 2015;372:1179-81.

10. Steck N, Egger M, Maessen M, et al. Euthanasia and assisted suicide in selected European countries and US states: systematic literature review. Med Care 2013; 51:938-44.

11. Smets T, Bilsen J, Cohen J, et al. Legal euthanasia in Belgium: characteristics of all reported euthanasia cases. Med Care 2010;48:187-92.

12. Deaths, estimates, by province and territory, CANSIM [Table 051-0004 and Cat. 91-215-X]. Ottawa: Statistics Canada; 2015.

13. The Way Forward Integration Initiative. Cost-effectiveness of palliative care: $a$ review of the literature. Ottawa: Canadian Hospice Palliative Care Association.

14. Bill C-14. An Act to amend the Criminal Code and to make related amendments to other Acts, (medical assistance in dying), 1st session, 32nd Parliament, Ontario; 2016.

15. Schedule of benefits for physician services under the Health Insurance Act. Toronto: Ministry of Health and Long-Term Care; 2015. Available: www.health.gov.on.ca/ english/providers/program/ohip/sob/physserv/physserv_mn.html (accessed 2016 Aug. 15).

16. Pharmacist (AHS recruitment). Edmonton: Alberta Health Services. Available: www.albertahealthservices.ca/careers/Page11743.aspx (accessed 2016 Aug. 15).

17. Snijdewind MC, Willems DL, Deliens L, et al. A study of the first year of the endof-life clinic for physician-assisted dying in the Netherlands. JAMA Intern Med 2015;175:1633-40.

18. Dierickx S, Deliens L, Cohen J, et al. Comparison of the expression and granting of requests for euthanasia in Belgium in 2007 vs 2013. JAMA Intern Med 2015; 175:1703-6.

19. Euthanasia, assisted suicide and non-resuscitation on request. The Hague (the Netherlands): Government of the Netherlands. Available: https://www. government.nl/topics/euthanasia/contents/euthanasia-assisted-suicide-and-non -resuscitation-on-request (accessed 2016 Apr. 2).

20. Raus K. The extension of Belgium's euthanasia law to include competent minors. J Bioeth Inq 2016;13:305-15.

21. Canadian Medical Association draft policy document. Principles-based approach to assisted dying in Canada. Canadian Medical Association; 2015. Available: www.cma.ca/Assets/assets-library/document/en/advocacy/EOL/ care-at-the-end-of-life-cma-framework-june2015-e.pdf (accessed 2015 Dec. 12).
Competing interests: Braden Manns reports receiving grants from Baxter Canada outside of the submitted work. No other competing interests were declared.

This article has been peer reviewed.

Accepted: Sept. 20, 2016

Affiliations: Departments of Medicine and Community Health Sciences, Cumming School of Medicine (Trachtenberg); O'Brien Institute of Public Health and Libin Institute for Cardiovascular Health (Trachtenberg, Manns); University of Calgary, Calgary, Alta.

Contributors: Aaron Trachtenberg developed the study idea, conducted all analyses, wrote the initial draft and provided critical revisions. Braden Manns provided key inputs into the protocol, supervised analyses and provided key revisions to the study protocol. Aaron Trachtenberg and Braden Manns read and approved the final manuscript and agreed to act as guarantors of the work.
Disclaimer: This study was supported by data from the Institute for Clinical Evaluative Sciences (ICES), which is funded by an annual grant from the Ontario Ministry of Health and Long-Term Care (MOHLTC). The opinions, results and conclusions reported in this paper are those of the authors and are independent from the funding sources. No endorsement by ICES or the Ontario MOHLTC is intended or should be inferred.

Acknowledgements: The authors thank Peter Tanuseputro and Mathieu Chalifoux for providing us with the end-of-life cost data essential to our model, Flora Au for assistance in developing the model, and Sarah Gil for figure preparation and manuscript formatting. Braden Manns is supported by the Svare Chair in Health Economics, an Alberta Innovates Health Scholar award, and a Canadian Insitutes of Health Research Foundation Award.

Correspondence to: Braden Manns, braden.manns@ albertahealthservices.ca 